Linköping Studies in Science and Technology. Thesis No. 1903 Licentiate Thesis

\title{
Performance Analysis in Wireless HetNets: Traffic, \\ Energy, and Secrecy Considerations
}

Georgios Smpokos 



\title{
Performance Analysis in Wireless HetNets: Traffic, Energy, and Secrecy Considerations
}

\author{
Georgios Smpokos
}

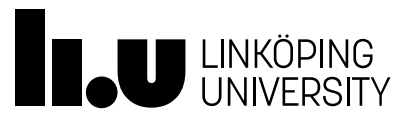

Department of Science and Technology

Linköping University, SE-601 74 Norrköping, Sweden 
(oc) EY-No This work is licensed under a Creative Commons AttributionNonCommercial 4.0 International License.

https://creativecommons.org/licenses/by-nc/4.0/

Performance Analysis in Wireless HetNets: Traffic, Energy, and Secrecy Considerations

Georgios Smpokos

LIU-TEK-LIC 2021

ISBN 978-91-7929-669-8

ISSN $0280-7971$

Linköping University

Department of Science and Technology

SE-601 74 Norrköping

Printed by LiU Tryck, Linköping, Sweden 2021 


\section{Abstract}

To this day, most of the communication networks are characterized by a "monolithic" operating approach. Network elements are configured and operate without any reconfiguration for long time periods. Softwarization, whereby dedicated elements are being replaced by more general-purpose devices, has been lately challenging this existing approach. Virtualizing the infrastructure through the softwarization can provide significant benefits to end users and operators, supporting more flexible service deployment, providing real time monitoring and operational changes.

In this licentiate thesis, we consider techniques that can be used towards virtual networking. In Paper I we study a novel allocation technique and traffic optimization process for the access network. Cellular network technologies (i.e. UMTS, LTE, LTE-A) will coexist with non-cellular small cells and offload traffic from cellular to non-cellular networks mainly operating in 3GPP Wi-Fi (IEEE 802.11 standards). This is a scenario for indoor wireless access implementations where offloading mechanisms can improve the QoS offered by the operators, and reduce the traffic handled by the access fronthaul. The analysis of a novel optimization algorithm exhibited a holistic solution for access-core interworking where LWA (LTE-WiFi Aggregation) offers improved performance for the end users.

In order to optimize core network operations factors such as the operational costs should be addressed. Following this approach in Paper II we analysed how environmental factors (e.g. temperature, humidity) can affect the power consumption of core network data centers (cooling systems). By applying machine learning techniques using data from a data center, we were able to forecast the power consumption based on to atmospheric weather conditions and analyse its accuracy.

Optimizing the access network operations and the interworking (resource allocation, scheduling, offloading) can lead to highly configurable and secure operations. These have been factors of great concern as wireless connectivity increases in denser populated areas. In Paper III we examine the physical layer secrecy aspects of a collaborative small cell network in the presence of parallel connections and caching capabilities at the edge nodes. Using tools from the probability theory, we examined how the power allocation for the transmissions can ensure secrecy in the presence of an eavesdropper. 



\section{Acknowledgments}

First and foremost, I would like to thank my academic supervisors Associate Professors Vangelis Angelakis and Nikolaos Pappas for their support and guidance during my studies at the Linköping University. Their dedication and drive for research and science have turned my $\mathrm{PhD}$ studies into a productive and stimulating process.

I would like to express my sincere gratitude to my industrial supervisor Dr. Athanasios Lioumpas. His input and guidance were always invaluable, especially the research related to the industry.

Moreover, I acknowledge all the members of the WiVi-2020 project, and especially Dr. Theodoros Mouroutis for giving me the opportunity to enroll in the industrial placement in CYTA Hellas and my PhD studies in Linköping University and make a smooth transition from the United Kingdom to Greece. My research was supported by the Europen Union's Horizon 2020 Marie Skłodowska-Curie Actions project WiVi-2020 (H2020-MSCA-ITN-2014-EID 642743-WiVi2020).

I thank my fellow WiVi-2020 researchers in LiU ad UoC: Antzela Kosta, Nader Daneshfar and Mohamed Elshatshat for the stimulating discussions, and the assistance they provided in these years.

Finally, I would like to thank the large community of researchers in LiU's Norrköping campus who welcomed me and assisted me enormously. Yanni. P, Mano, Niko, Yanni A., Maria, Elina, Cristian thank you for all your support.

Last but not least, I would like to thank my companion in life Maria for being always there to encourage me in this journey. This work is dedicated to her and our precious Olga.

Athens, February 2021

Georgios Smpokos 



\section{Abbreviations}

5G Fifth Generation

AP Access Point

API Application Programming Interface

BS Base Station

CAPEX Capital Expenditure

CN Core Network

CSI Channel State Information

DC Data Center

eMBB enhanced Mobile Broadband

eNB evolved node B

EP Equilibrium Point

EPC Enhanced Packet Core

HetNet Heterogeneous Network

ICIC Inter-Cell Interference Coordination

IoT Internet of Things

LAN Local Area Network

LTE Long Term Evolution

LWA LTE-WiFi Aggregation 
MEC Multi-access Edge Computing

MIMO Multiple-Input Multiple-Output

ML Machine Learning

mMTC massive Machine Type Communications

MNO Mobile Network Operator

MTC Machine Type Communication

MVNO Mobile Virtual Network Operator

NAS Non Access Stratum

NF Network Function

NFV Network Function Virtualization

OFDMA Orthogonal Frequency Division Multiple Access

OPEX Operational Expenditure

PGW Primary Gateway

PHY Physical Layer

QoE Quality of Experience

QoS Quality of Service

RACH Radio Access Channel

RAN Radio Access Network

RAT Radio Access Technology

RTD Round-trip Delay

SDN Software Defined Networking

SGW Secondary Gateway

SIA Service to Interference Assignment

TOTFA Traffic Offloading and Transmission Time Fraction Allocation 
UE User Equipment

VLAN Virtual Local Area Network

VM Virtual Machine

VNF Virtual Network Function

VPN Virtual Private Network

VR Virtual Resource

WiFi Wireless Fidelity

WLAN Wireless Local Area Network

WVN Wireless Virtualized Network

WSP Wireless Service Provider 



\section{Contents}

Abstract

Acknowledgments

Abbreviations

vii

I Introduction $\quad 1$

1 Network Virtualization 3

2 Optimization of Core-Access Networks $\quad 7$

3 Publications and Contributions 10

Bibliography 13

$\begin{array}{ll}\text { II Papers } & 19\end{array}$

$\begin{array}{ll}\text { Paper I } & 21\end{array}$

Paper II 39

$\begin{array}{ll}\text { Paper III } & 61\end{array}$ 



\section{Part I}

\section{Introduction}





\section{Network Virtualization}

In recent years there has been an increasing demand for high throughput, low latency, energy efficiency, security and connectivity prompted by massive numbers of connected multipurpose devices, Internet of Things (IoT). The ongoing explosion in connectivity requirements and the increasing demand for real-time services e.g., video streaming and live conferencing, are stretching the already limited capacity of the deployed network systems and operations. All these requirements have paved the way to a new era for communication standards and deployments, leading to the Fifth Generation (5G) of networks, affecting both research and standardization [1-3].

New Physical Layer (PHY) methods for the $5 \mathrm{G}$ standards have already been proposed and tested including Multiple-Input MultipleOutput (MIMO) systems, full duplex scenarios, and modulation schemes. Novel physical layer technologies such as Inter-Cell Interference Coordination (ICIC), will lead to an increased spectrum efficiency as more Heterogeneous Network (HetNet) implementations will offer the required services to the subscribers $[4,5]$. HetNets used for optimizing user experience and reducing the cellular network traffic have been examined and will eventually lead to more efficient resource allocation for multiple services utilizing a variety of access technologies [5-8].

Currently, the dedicated hardware-based core and access network components cannot provide the necessary flexibility and efficiency at the control plane (switches, gateways, controllers). Due to these factors, a new backhaul network core-access architecture has to be adopted based on Software Defined Networking (SDN) and Network Function Virtualization (NFV) paradigms [9-12].

\subsection{Resource Allocation and Scheduling}

As we are entering the IoT interconnected era, next generation networks need to support heterogeneous services with diverse specifications. These specifications should lead to a network operation where resources, both physical and virtual, are being efficiently allocated and scheduled to the users. In [16] the authors have tried to optimize the mechanism of allocating Virtual Resources (VR's) based on multiple application demands at the user end, introducing two different algorithms for resource allocation. The users could be served by 
more than one interface (Radio Access Network (RAN) technology) such as Long Term Evolution (LTE), IEEE 802.11.x (Wireless Fidelity (WiFi)). The purpose of the optimization was to minimize the total utilization cost of each interface's resources based on user demands namely the Service to Interference Assignment (SIA) problem. Additionally, the authors of [17] suggested a novel optimization of resource allocation approach using Linear Programming and the Lagrangian duality theory. The results demonstrated great advantages when adopting a flexible allocation strategy in time and frequency domains, eventually increasing the utilization of network capacity for mission critical services. Based on the concept of LTE-WiFi Aggregation (LWA), the authors of [18] studied an LWA-enabled network that included an LTE Base Station (BS) and a Wi-Fi Access Point (AP) where a non-ideal backhaul affected the LWA design parameters such as throughput and delay performance.

Backhaul capacity improvements and denser deployments of HetNet's will eventually increase resource availability and enhance the end to end Quality of Service (QoS). Motivated by this, the authors of [19] presented a scheduling mechanism within a two-tier HetNet deployment, where traffic offloaded to femto-cell BS's is served by the core network via residential broadband connections. In this constrained backhaul capacity scenario, the implementation of Traffic Offloading and Transmission Time Fraction Allocation (TOTFA) was examined while proportional fairness regarding throughput among users was applied.

Fairness among users for resource allocation is an important concept that can be applied for high density and multi-purpose multiservice network implementations. Fair traffic allocation and aggregation in HetNet's is of a great significance as more network operators utilize low power Wireless Local Area Network (WLAN) femtocells where traffic is offloaded from LTE or other next generation macro cell Radio Access Technologies (RAT's). Following these, in [20] the authors have proposed an algorithmic solution for splitting traffic in LTE-WLAN HetNet's based on LWA while experiencing some network backhaul delay and maximizing the average User Equipment (UE) throughput performance. By implementing a water filling technique for allocating macro cell resources, the proposed mechanism performs better than other RAT selection algorithms that had been proposed and implemented. This work has been the foundation of our study in Paper I where the concept of slicing (grouping the end 
users) could lead to an improved resource scheduling mechanism.

A market oriented problem of allocating VR's where Mobile Network Operators (MNO's) offer their VR's to Wireless Service Providers (WSP's) while maximizing the utilization within a Wireless Virtualized Network (WVN) is studied in [21]. The authors of this work proposed an analytical solution for allocating Orthogonal Frequency Division Multiple Access (OFDMA) orthogonal subcarriers (spectrum allocation), eventually reaching an Equilibrium Point (EP) where both the MNO and the WSP maximize their profits. This maximization of the utilization of the available VR's takes into consideration the quality of the channel (Channel State Information (CSI)) extracted from the subscribers. The algorithm used to solve the problem converges, performing a per resource price adjustment based on the supply and demand values of VR's.

\subsection{Software Defined Networking}

Following the paradigm of Data Centers (DC's) and Local Area Networks (LAN's) where virtualization (Virtual Local Area Network (VLAN), Virtual Private Network (VPN)) has been widely deployed, both the network elements and services will be transformed to virtual commodities. This transformation will reduce the operation and deployment costs, centralize the control, and become more susceptible to future upgrades. SDN technologies aim to drive this implementation of virtual networks, more specifically by decoupling network control from the forwarding functions, thus decoupling control and data planes $[10,22]$.

In [9] the authors explicitly analysed the key features that $5 \mathrm{G}$ technologies and networks should support, such as increased traffic volumes, high user data rates, low latency and supporting a high number of Machine Type Communication (MTC) devices in the IoT framework. The need for power efficiency at the user end was considered as well as how the reduced operation costs could benefit a Mobile Virtual Network Operator (MVNO) operating a virtual network. Notably, the proposed architecture introduces the notions of SDN and NFV simultaneously to the RAN and Core Network (CN) making the entire network highly programmable, scalable, and ready for the virtualization of its components.

Bottlenecks for applying virtualization at the $\mathrm{CN}$ have been extensively studied in [15]. In this work the authors studied the perfor- 
mance of Enhanced Packet Core (EPC) components of the backhaul network being operated in a virtual environment. Furthermore, this research focused on defining the impact of control plane misbehaviour in user plane data flow performance. The model used for this research was based on real life traffic data provided by an MNO. The number of control plane events were critical in managing and processing the traffic of the data plane. After the detailed analysis of Non Access Stratum (NAS) events, the authors concluded that the Secondary Gateway (SGW) is crucial in terms of control plane signalling but also in data-user plane traffic flow and thus should be taken into consideration when transforming the hardware into virtual functions. In [23], the Virtual Network Function (VNF) placement and its implementation was extended to the RAN and a novel VNF placement algorithm was studied. In this problem's definition the authors took into consideration forwarding and processing characteristics of the network nodes as well as the capacity of the virtual interconnection links. The proposed VNF placement heuristic could eventually allow MVNO's that do not own any physical CN or RAN resources to implement resource allocation for service provisioning and delivery.

HetNet Cloud infrastructure as part of an SDN framework of HetNets has been extensively examined in [24] where the authors emulated a scenario using Mininet simulations tool. In that work the research was focused on a detailed overview of an end-to-end virtualized infrastructure where control plane entities reside in servers decoupled from any hardware middleboxes. Southbound and Northbound Application Programming Interfaces (API's) were presented and QoS constraints such as Round-trip Delay (RTD) affecting services such as live voice and video sharing have been examined. That work identified that topology for the placement of Virtual Machines (VM's) on the physical substrate must be very carefully examined and planned in any HetNet SDN implementation. In [11] the authors applied NFV and offloading to Multi-access Edge Computing (MEC) servers in order to study the end-to-end service performance of a communication system. Through that analysis they derived approximate analytical expressions for the end-to-end delay, throughput, and drop rate. 


\section{Optimization of Core-Access Networks}

The technologies covered in the previous section enable flexibility through the softwarization of the network components as well through separating the control and data planes (network subsystems control and user plane for data transmission) eventually offering upgradability, optimization, and customization of networking [4]. For a network that will host users with very different and versatile needs and requirements in terms of throughput, latency, availability, and security, the MNO's and MVNO's need to classify their subscribers into user groups. This separation will be based on user requirements and will assist in providing advanced services through the creation of virtual network slices, reducing the signalling, control overhead, and optimizing the coordination of core with RAN interfaces and components $[13,14]$.

The centralized management controllers of the network will coordinate physical and virtualized processes such as routing, spectrum allocation, power efficiency, and caching, to name a few. These managements tasks need to be further investigated while novel optimization algorithms should be introduced to cope with the excess of data traffic in various HetNet implementations [9]. In order to achieve the aforementioned goals, the bottlenecks of virtualizing the core and access network functionality needs to be evaluated and thoroughly examined [15].

\subsection{Network Slicing}

In the context of offering multiple services with diverse specifications (video, voice, real-time and reliable communications) and to multipurpose devices (IoT, automotive, smartphones), network operators should define and implement end to end slicing. Slicing the network will offer isolation, functional, and performance independency and security in both core and access levels [13, 14, 22, 23].

In [13], a flexible architecture implementing network slicing demonstrated how different functions could be controlled and be set effectively for end to end services. The authors pointed out the importance of having a flexible realization of end to end slices and proposed a selection-attachment mechanism for users to further reduce control signalling. That scenario included two different network slices dedicated to two user groups namely enhanced Mobile Broadband (eMBB) 
and massive Machine Type Communications (mMTC) users [26]. It was emphasized that some of the core Network Functions (NF's) can be migrated to the access-RAN domain as this will reduce the signalling traffic and increase the Quality of Experience (QoE) offered to the end to end slice members. By applying slice registration requests and selection at the access network entities, operators could achieve less attachment delays enabling faster slice access by introducing a dedicated Radio Access Channel (RACH) slice.

In [14] a holistic mobile packet core network implementation on SDN and NFV was proposed namely the V-Core. This approach introduced an SDN controller that could define the slicing and services provisioned per user plus separate the $\mathrm{CN}$ into different control planes operated by different MVNO's. After analysing the network topology for V-Core the authors illustrated the layered (control, data) structure of data flows indicating that not only the control plane could reside on cloud-virtual infrastructure. Additionally, the data plane functions (SGW and Primary Gateway (PGW) for LTE networks) could be implemented in a similar fashion. That study has finally indicated the cost efficiency of this implementation by significantly reducing Capital Expenditure (CAPEX) and Operational Expenditure (OPEX) compared to old fashioned hardware based network implementations.

\subsection{Energy Efficiency}

In order for the network operators to reduce their OPEX, energy consumption and efficiency could become one of these elements to be optimized. Both in access and core networks energy consumption has greatly affected the operational costs while driving high power amplifiers at the edge of cellular networks or hosting core elements and servers in DC's [27-30]. As caching will be deployed at the edge of the networks and not solely at the CN, energy efficiency can be highlighted as one of the main elements to be optimized regarding the operations. Especially within DC's, a big portion of the power consumption comes from the cooling systems operated to control the temperature levels within them [31].

Patterns of energy consumption can be traced and identified similarly to traffic patterns indicating higher demand at specific periods of time. Making the energy consumption of multiple network elements more efficient will enable a greener operation of the network. Predict- 
ing the energy consumption could eventually lead to a better usage of energy generated by renewable resources. Identifying these patterns can be realized using data from the operation and some preliminary data analysis. Moving further, predictions on the consumption can be performed using machine learning techniques where models can be used to forecast energy requirements e.g. in DC's their energy consumption [32-35].

\subsection{Security and Caching}

Another significant factor affecting the overall operations of the MNO's has been the security of their networks. Although fiber optics had offered a secure transmission down to the last mile of edge networks, the physical layer (PHY) security of wireless access networks needs to be further examined and studied. Studies have proved that secure physical layer wireless communications can be established over noisy channels and fading conditions and enable secrecy and reliability for the connectivity [36-45].

In order for small cells to establish and utilize in terms of stability and security multiple connections, caching needs to be available at the edge nodes [46, 47]. Caching popular content at specific nodes could also assist in reducing latency and processing within the network. This can be achieved evolving the RAN data plane where $5 \mathrm{G}$ evolved node B (eNB)-base stations will be able to split the protocol and hardware functionality, will be programmable to support multiple RAT's and support caching at the edge.

Caching could eventually improve the stability of networks by providing the most popular content to users that are served in a specific area by storing it in edge caches $[48,51]$. Storing content at the edge nodes can offload a significant amount of traffic from CN's, improve the stability of the overall network and offer high QoE to the end users. Specific caching scenarios with different KPI's have been examined and optimized, such as the cache hit ratio, the cache-aided throughput [52], and the energy efficiency [53]. In [54] a cooperation setup has been proposed that minimizes the average energy consumption of a UE in order to receive its requested content. The QoE for edge users is studied in [55] where a novel algorithm is proved to optimize the user QoE and increase network performance by applying Machine Learning (ML) techniques. 


\section{Publications and Contributions}

The respective research items, dealing with i) an optimal algorithm implementation for resource allocation in heterogeneous access network, ii) the energy consumption forecasting based on machine learning techniques, and iii) physical layer secrecy for access networks with caching capabilities are presented in the following sections.

The main contributions are summarized as follows:

- Optimal resource allocation algorithm implementation for the access network for reducing users' average delay performance.

- Allocating resources to slices of users satisfying throughput and delay requirements.

- Data analysis and correlation of energy consumption in core data centers with external weather conditions using machine learning techniques.

- Identifying the effects of caching on the performance of a system under different traffic characteristics.

- The analysis of the impact of physical layer secrecy on the delay and throughput performance of heterogeneous networks.

The research papers are summarized below:

Paper I: Performance Aware Resource Allocation and Traffic Aggregation for User Slices in Wireless HetNets, co-authored with Athanasios Lioumpas, Theodoros Mouroutis, Yiannis Stylianou, and Vangelis Angelakis. The paper has been published in Proc. of IEEE 22nd International Workshop on Computer Aided Modelling and Design of Communication Links and Networks (CAMAD), June 2017.

In Paper I, the performance of an optimal resource allocation algorithm is examined while adding a new delay aware process aiming in jointly achieving predefined throughput and delay performance for selected groups/slices of users. From the optimal resource allocation, an algorithmic solution was derived which can be applied to determine an optimal number of network slices to be served. Determining 
how to jointly assign spectrum resources and power for these optimization problems by using discrete rate levels and discrete power levels is investigated.

Paper II: On the Energy Consumption Forecasting of Data Centers Based on Weather Conditions: Remote Sensing and Machine Learning Approach, co-authored with Mahamed Elshatashat, Athanasios Lioumpas, and Ilias Iliopoulos. The paper has been published in Proc. of IEEE 11th International Symposium on Communication Systems, Networks 8 Digital Signal Processing (CSNDSP), September 2018.

Paper II exploits the data provided by the FIESTA-IoT platform [56] in order to investigate the correlation between the weather conditions and the energy consumption in a data center. Using multivariable linear regression, correlation between the energy consumption and the dominant weather condition parameters is modelled in order to effectively forecast the energy consumption based on the weather forecast. The machine learning technique used in this research utilised a backward elimination mechanism to extract the most significant independent parameters affecting the power consumption.

Paper III: Performance Analysis of a Cache-Aided Wireless Heterogeneous Network with Secrecy Constraints, coauthored with Zheng Chen, Parthajit Mohapatra, and Nikolaos Pappas. The paper has been submitted in IEEE Access, January 2021.

Paper III deals with the investigation and analysis of the performance of a wireless system with caching capabilities while imposing secrecy constraints at one of the users being served. In this paper the system model where an eavesdropper is introduced and the caching characteristics were deployed and defined. Next, the analysis based on queing theory was introduced and the closed forms for throughput and delay performance were extracted. Two distinct demodulation schemes were analysed and results were produced for the comparisson. The effects of caching on the secrecy of the system was examined and two demodulation schems were compared. Finally, two optimization problems were set in order to get optimal setting values for this schenario and achieve maxium throughput and minimum delay values for each user. 



\section{Bibliography}

[1] EU Digital Economy \& Society Commission, "5G Manifesto for timely deployment of 5G in Europe," 5G Manifesto, Jul. 2016.

[2] Cisco, "Cisco visual networking index: Global mobile data traffic forecast update 2014-2019," White Paper, Feb. 2015.

[3] O. Holland et al., "The IEEE 1918.1 "Tactile Internet" Standards Working Group and its Standards," in Proceedings of the IEEE, vol. 107, no. 2, pp. 256-279, Feb. 2019.

[4] M. Jaber, M. Imran, R. Tafazolli, and A. Tukmanov, "5G Backhaul Challenges and Emerging Research Directions: A Survey", IEEE Access, vol. 4, no. 3, pp. 1743-1766, Apr. 2016.

[5] C. X. Wan et al, "Cellular Architecture and Key Technologies for 5G Wireless Communication Networks", IEEE Communications Magazine, vol. 52, no. 2, pp. 122-130, Feb. 2014.

[6] M. Gerasimenko et al, "Cooperative Radio Resource Management in Heterogeneous Cloud Radio Access Networks", IEEE Access, vol. 3, pp. 397-406, Apr. 2015.

[7] S. Borst, S. Hanly, and P. Whiting, "Optimal Resource Allocation in HetNets", IEEE International Conference on Communications (ICC), Jun. 2013.

[8] Z. Chen and M. Kountouris, "Guard zone based D2D underlaid cellular networks with two-tier dependence," 2015 IEEE International Conference on Communication Workshop (ICCW), London, pp. 222-227, Jun. 2015. 
[9] J. Zhang, W. Xie, and F. Yiang, "An Architecture for 5G Mobile Network Based on SDN and NFV", IET 6th International Conference on Wireless, Mobile and Multi-Media (ICWMMN 2015), Nov. 2015.

[10] Q. Zhou, C. X. Wang, S. McLaughlin, and X. Zhou, "Network Virtualization and Resource Description in Software-Defined Wireless Networks", IEEE Communications Magazine, vol. 53, no. 11, pp. 110-117, Nov. 2015.

[11] E. Fountoulakis, Qi Liao, N. Pappas, "An End-to-End Performance Analysis for Service Chaining in a Virtualized Network", IEEE Open Journal of Communications Society, vol. 1, pp. 148$163,2020$.

[12] I. Avgouleas, D. Yuan, N. Pappas, V. Angelakis, "Virtual Network Functions Scheduling under Delay-Weighted Pricing", IEEE Networking Letters, vol. 1, no. 4, Dec. 2019.

[13] X. An et al, "On end to end network slicing for 5G communication systems", Transactions on Emerging Telecommunications Technologies, Wiley Online Library 2016.

[14] V. G. Nguen, and Y. H. Kim, "Slicing the Next Mobile Packet Core Network", IEEE 11th International Symposium on Wireless Communications Systems (ISWCS), Aug. 2014.

[15] A. S. Rajan et al, "Understanding the bottlenecks in Virtualizing Cellular Core Network Functions", IEEE 21st IEEE International Workshop on Local and Metropolitan Area Networks, Apr. 2015.

[16] V. Angelakis et al, "Allocation of Heterogeneous Resources of an IoT Device to Flexible Services", IEEE Internet of Things Journal, vol. 3, no. 5, pp. 691-700, Oct. 2016.

[17] L. You, Q. Liao, N. Pappas and D. Yuan, "Resource Optimization With Flexible Numerology and Frame Structure for Heterogeneous Services," IEEE Communications Letters, vol. 22, no. 12, pp. 2579-2582, Dec. 2018.

[18] B. Chen, N. Pappas, Z. Chen, D. Yuan, J. Zhang, "Throughput and Delay Analysis of LWA with Bursty Traffic and Randomized Flow Splitting", IEEE Access, vol. 7, 2019. 
[19] P.-Y. Kong, and G. K. Karagiannidis, "Backhaul-Aware Joint Traffic Offloading and Time Fraction Allocation for 5G HetNets", IEEE Transactions on Vehicular Technology, vol. 65, no. 11, pp. 9224-9235, Nov. 2016.

[20] S. Singh et al, "Proportional Fair Traffic Splitting and Aggregation in Heterogeneous Wireless Networks", IEEE Communications Letters, vol. 20, no. 5, pp. 1010-1013, Mar. 2016.

[21] G. Zhang et al, "Virtual Resource Allocation for Wireless Virtualization Networks Using Market Equilibrium Theory", IEEE Conference on Computer Communications Workshops (INFOCOM WKSHPS), May 2015.

[22] C. Liang, and F. R. Yu, "Wireless Virtualization for Next Generation Mobile Cellular Networks", IEEE Wireless Communications, vol. 22, no. 1, pp. 61-69, Feb. 2015.

[23] R. Riggio et al, "Scheduling Wireless Virtual Networks Functions", IEEE Transactions on Network and Service Management, vol. 13, no. 2, pp. 240-252, Jun. 2016.

[24] M. M. Rahman, C. Despins, and S. Affens, "Service Differentiation in Software Defined Virtual Heterogeneous Wireless Networks", 2015 IEEE International Conference on Ubiquitous Wireless Broadband (ICUWB), Oct. 2015.

[25] E. Fountoulakis, Qi Liao, N. Pappas, "An End-to-End Performance Analysis for Service Chaining in a Virtualized Network", IEEE Open Journal of Communications Society, vol. 1, pp. 148$163,2020$.

[26] ITU-R, "IMT Vision - Framework and overall objective of the future development of IMT for 2020 and beyond", in ITU - M Series, Sep. 2015.

[27] Google, "Google data centres, efficiency: How we do it", www.google.com/about/datacenters/efficiency/.

[28] J. Cho, T. Lim, and B. Kim, "Viability of data centre cooling systems for energy efficiency in temperate or subtropical regions: Case study", Journal of Energy and Buildings, vol. 55, pp. 189-197, Dec. 2012. 
[29] BBC, "Inside Facebook's green and clean arctic data centre", http://www.bbc.com/news/business-22879160, Jun. 2013.

[30] A. Mousavi, Y. Berezovskaya, V. Vyatkin, X. Zhang, and T. Minde, "Improvement of energy efficiency in data centers via flexible humidity control", IECON 2016, Oct. 2016.

[31] M. Dayarathna, Y. Wen, and R. Fan, "Data center energy consumption modeling: A survey", IEEE Communications Surveys Tutorials, Sep. 2016.

[32] Q. Zeng et al., "An optimum regression approach for analyzing weather influence on the energy consumption", International Conference on Probabilistic Methods Applied to Power Systems (PMAPS), Oct. 2016.

[33] M. Torabi and S. Hashemi, "A data mining paradigm to forecast weather sensitive short-term energy consumption", AISP, May 2012 .

[34] Y. Foo, C. Goh, H. Lim, Z. Zhan, and Y. Li, "Evolutionary neural network based energy consumption forecast for cloud computing", International Conference on Cloud Computing Research and Innovation (ICCCRI), Oct. 2015.

[35] A. Ahmad et al., "A review on applications of ann and svm for building electrical energy consumption forecasting", Renewable and Sustainable Energy Reviews, vol. 33, pp. 102 - 109, May 2014.

[36] I. Csiszar and J. Korner, "Broadcast channels with confidential messages", IEEE Trans. Inf. Theory, vol. IT-24, no. 3, pp. 339348, May 1978.

[37] E. Ekrem and S. Ulukus, "Secrecy in cooperative relay broadcast channels", IEEE Trans. Inf. Theory, vol. 57, no. 1, pp. 137-155, Jan. 2011.

[38] R. Liu, I. Maric, P. Spasojevic, and R. Yates, "Discrete memoryless interference and broadcast channels with confidential messages: Secrecy rate regions", IEEE Trans. Inf. Theory, vol. 54, no. 6, pp. 2493-2507, Jun. 2008.

[39] O. Koyluoglu and H. El Gamal, "Cooperative encoding for secrecy in interference channels", IEEE Trans. Inf. Theory, vol. 57, no. 9, pp. 5682-5694, Sep. 2011. 
[40] E. Ekrem and S. Ulukus, "Effects of cooperation on the secrecy of multiple access channels with generalized feedback", CISS, Mar. 2008.

[41] A. Khisti, A. Tchamkerten, and G. W. Wornell, "Secure broadcasting over fading channels", IEEE Trans. Inf. Theory, vol. 54, no. 6, pp. 2453-2469, Jun. 2008.

[42] J. Barros and M. R. D. Rodrigues, "Secrecy capacity of wireless channels", Proc. IEEE Int. Symp. Inf. Theory, pp. 356-360, Jul. 2006 .

[43] P. K. Gopala, L. Lai and H. El Gamal, "On the Secrecy Capacity of Fading Channels", IEEE Trans. Inf. Theory, vol. 54, no. 10, pp. 4687-4698, Oct. 2008.

[44] K. Jiang, T. Jing, Z. Li, Y. Huo and F. Zhang, "Analysis of secrecy performance in fading multiple access wiretap channel with SIC receiver", IEEE INFOCOM, May 2017.

[45] P. Mohapatra, N. Pappas, J. Lee, T. Q. S. Quek, V. Angelakis, "Secure Communications for the Two-user Broadcast Channel with Random Traffic", IEEE Transactions on Information Forensics and Security, vol. 13, no. 9, Sep. 2018.

[46] F. Rezaei and B. H. Khalaj, "Stability, rate, and delay analysis of single bottleneck caching networks", IEEE Trans. Commun., vol. 64, no. 1, pp. 300-313, Jan. 2016.

[47] N. Pappas, Z. Chen and I. Dimitriou, "Throughput and delay analysis of wireless caching helper systems with random availability", IEEE Access, vol. 6, pp. 9667-9678, 2018.

[48] K. Shanmugam, N. Golrezaei, A. G. Dimakis, A. F. Molisch, and G. Caire, "FemtoCaching: Wireless content delivery through distributed caching helpers", IEEE Trans. Inf. Theory, vol. 59, no. 12, pp. 8402-8413, Dec. 2013.

[49] G. Paschos, E. Bastug, I. Land, G. Caire, and M. Debbah, "Wireless caching: Technical misconceptions and business barriers", IEEE Commun. Mag., vol. 54, no. 8, pp. 16-22, Aug. 2016.

[50] N. Carlsson and D. Eager, "Ephemeral content popularity at the edge and implications for on-demand caching", IEEE Trans. Parallel Distrib. Syst., vol. 28, no. 6, pp. 1621-1634, Jun. 2017. 
[51] Z. Chen and M. Kountouris, "D2D caching vs. small cell caching: Where to cache content in a wireless network?", in Proc. IEEE 17th Int. Workshop Signal Process. Adv. Wireless Commun. (SPAWC), pp. 1-6, Jul. 2016.

[52] Z. Chen, N. Pappas, and M. Kountouris, "Probabilistic caching in wireless D2D networks: Cache hit optimal versus throughput optimal", IEEE Commun. Lett., vol. 21, no. 3, pp. 584-587, Mar. 2017.

[53] D. Liu and C.Yang, "Energy effciency of downlink networks with caching at base stations", IEEE J. Sel. Areas Commun., vol. 34, no. 4, pp. 907-922, Apr. 2016.

[54] J. Ma, J. Wang, and P. Fan, "A cooperation-based caching scheme for heterogeneous networks", IEEE Access, vol. 6, pp. 15013-15020, 2017.

[55] S. M. S. Tanzil, W. Hoiles, and V. Krishnamurthy, "Adaptive scheme for caching YouTube content in a cellular network: Machine learning approach", IEEE Access, vol. 5, pp. 5870-5881, 2017.

[56] Federated Interoperable Semantic IoT Testbeds and Applications (FIESTA-IoT) / Cyta Hellas, "DC-IoT: Monitoring Energy Efficiency for Data Centres", http://fiestaiot.eu/index.php/fiesta-experiments/dc-iot-monitoring-energyefficiency-for-data-centres/, 2018. 


\section{Part II}

\section{Papers}





\section{Papers}

The papers associated with this thesis have been removed for copyright reasons. For more details about these see:

http://urn.kb.se/resolve?urn=urn:nbn:se:liu:diva-174244 


\section{FACULTY OF SCIENCE AND ENGINEERING}

Linköping Studies in Science and Technology. Thesis No. 1903, 2021 Licentiate Thesis

Department of Science and Technology

Linköping University

SE-60174 Norrköping, Sweden

www.liu.se 